\title{
Significance of the Lymph Node Ratio in Stage III Colon Cancer
}

\author{
Simon H. Telian, $\mathrm{MD}^{1}$ and Anton J. Bilchik, MD, PhD, FACS ${ }^{2,3}$ \\ ${ }^{1}$ Division of Surgical Oncology, John Wayne Cancer Institute at Saint John's Health Center, 2200 Santa Monica Blvd., Santa \\ Monica, CA 90404, USA \\ ${ }^{2}$ California Oncology Research Institute, 2811 Wilshire Blvd., Suite 800, Santa Monica, CA 90403, USA \\ ${ }^{3}$ University of California at Los Angeles School of Medicine, Los Angeles, CA, USA
}

Last year, there was a great deal of attention devoted to lymph node status and the staging of colon cancer. This culminated in the National Quality Forum endorsing the 12-node minimum as a proxy measure of quality and necessary for adequate staging. This, however, has been criticized as an oversimplification because it is unclear whether lymph node evaluation is influenced by the surgeon, the pathologist, or even the patient. With more effective and expensive chemotherapeutic and biologic agents available for the treatment of colon cancer, there is a need to develop better staging methods to improve the selection of patients for therapy. Even in node-negative patients, the use of adjuvant chemotherapy is advocated by the American Society of Clinical Oncology solely on the basis of the low lymph node collection. Rather than number of lymph nodes alone as a prognostic factor, some have proposed lymph node ratio (LNR) also as an important measure.

Wang et al. ${ }^{1}$ report a retrospective study of data from the National Cancer Institute's Surveillance Epidemiology and End Results Program. They identified 24,477 patients with stage III colon cancer and examined the role of LNR in staging and prognosis. LNR, defined as the number of positive lymph nodes divided by the total number of lymph nodes examined, was an independent predictor for survival, which could be used to stratify patients with stage IIIB and IIIC colon cancer. Ratio groups LNR1

Published online March 25, 2008.

Address correspondence and reprint requests to: Anton J. Bilchik, MD, PhD, FACS; E-mail: abilchik@aol.com

Published by Springer Science+Business Media, LLC $\odot 2008$ The Society of Surgical Oncology, Inc. through LNR4 were defined as $<1 / 14,1 / 14$ to $25 \%$, $25 \%$ to $50 \%$, and $50 \%$ to $100 \%$, respectively. This study confirms the prognostic implications of LNR suggested in smaller retrospective studies ${ }^{2}$ and complements the reported correlation between more extensive nodal dissection and improved survival of patients with colon cancer. ${ }^{3}$

Although the tumor status of regional lymph nodes is essential for staging of colon cancer, the importance of the total number of collected lymph nodes remains controversial. A more extensive lymph node dissection was thought to increase survival only by increasing the likelihood of upstaging disease (i.e., higher likelihood of finding a disease-positive node when more lymph nodes are examined). However, further investigations have shown a direct correlation between number of collected nodes and clinical outcome within specific stages of disease. ${ }^{4}$ The reason for this difference in survival is still debatable. Interestingly, in the review by Wang et al., ${ }^{1}$ the prognostic significance of LNR was not dependent on the number of collected nodes. Of 9610 patients who had fewer than 10 lymph nodes examined, the survival difference between LNR2 $(\mathrm{N}=2888)$ and LNR4 $(\mathrm{N}=3734)$ was $22.4 \%(P<.0001)$.

Given the increased emphasis on implementing quality measures and documenting outcomes, the number of collected nodes is likely to become a factor in determining the quality of surgical procedures for colon cancer. Still, although the importance of an adequate lymphadenectomy by the surgeon cannot be overstated, it would be premature to propose adequacy of nodal resection as a quality indicator or criterion for reimbursement by insurance companies. 
As noted by Wang's group, ${ }^{1}$ the number of positive/total nodes also depends on the pathologist and the methods used to identify metastases in resected lymph nodes (i.e., hematoxylin and eosin staining, immunohistochemistry, or polymerase chain reaction). We have previously reported a marked increase in the detection of metastases by using sentinel node techniques in colon cancer. ${ }^{5}$ Collaboration between surgeon and pathologist is essential to ensure complete specimen evaluation.

Because LNR combines information on the number of disease-positive lymph nodes and the total number of resected nodes, both of which have independent prognostic significance, it merits further investigation as a possible standard tool for staging colon cancer.

\section{ACKNOWLEDGMENTS}

Supported by funding from the William Randolph Hearst Foundations (San Francisco, CA), the Davidow Charitable Fund (Los Angeles, CA), Mrs. Ruth Weil (Los Angeles, CA), the Harold J. McAlister
Charitable Foundation (Los Angeles, CA), the Family of Robert Novick (Los Angeles, CA), the Weil Family Fund (Los Angeles, CA), Rod Fasone Memorial Cancer Fund (Indianapolis, IN), and the Wrather Family Foundation (Los Alamos, CA).

\section{REFERENCES}

1. Wang J, Hassett JM, Dayton MT, et al. Lymph node ratio: role in the staging of node-positive colon cancer. Ann Surg Oncol 2008. DOI:10.1245/s10434-007-9716-x.

2. Schumacher P, Dineen S, Barnett C, et al. The metastatic lymph node ratio predicts survival in colon cancer. Am J Surg 2007; 194:827-32.

3. Chen SL, Bilchik AJ. More extensive nodal dissection improves survival for stages, I to III of colon cancer: a population based study. Ann Surg 2006; 244:602-10.

4. Johnson PM, Porter GA, Ricciardi R, et al. Increasing negative lymph node count is independently associated with improved long term survival in stage IIIB and IIIC colon cancer. J Clin Oncol 2006; 24:3570-5.

5. Bilchik AJ, Nora DT, Sobin LH, et al. Effect of lymphatic mapping on the new tumor-node-metastasis classification for colorectal cancer. J Clin Oncol 2003; 21:668-72. 